\title{
Revisiting surface core-level shifts for ionic compounds
}

\author{
Paul S. Bagus, ${ }_{1}^{1}$ Connie J. Nelin, ${ }^{2}$ Xunhua Zhao, ${ }^{3, *}$ Sergey V. Levchenko, ${ }^{3, \dagger}$ Earl Davis, ${ }^{4,+}$ Xuefei Weng, ${ }^{4,}{ }^{\S}$ Florian Späth, ${ }^{5}$ \\ Christian Papp, ${ }^{5}$ Helmut Kuhlenbeck $\odot,{ }^{4, \ddagger}, \mathbb{I}$ and Hans-Joachim Freund ${ }^{4}$ \\ ${ }^{1}$ Department of Chemistry, University of North Texas, Denton, Texas 76203-5017, USA \\ ${ }^{2} 6008$ Maury's Trail, Austin, Texas 78730, USA \\ ${ }^{3}$ Abteilung Theorie, Fritz-Haber-Institut der Max-Planck-Gesellschaft, Faradayweg 4-6, 14195 Berlin, Germany \\ ${ }^{4}$ Abteilung Chemische Physik, Fritz-Haber-Institut der Max-Planck-Gesellschaft, Faradayweg 4-6, 14195 Berlin, Germany \\ ${ }^{5}$ Lehrstuhl für Physikalische Chemie II, Universität Erlangen-Nürnberg, Egerlandstrasse 3, 91058 Erlangen, Germany
}

(Received 28 August 2019; published 12 September 2019)

\begin{abstract}
The shifts of core-level binding energies can provide powerful information about the electronic structure of a material. Understanding the physical origin of these shifts for catalytically relevant oxides may provide important insight into their properties. This requires reliable theoretical methods which are able to relate the binding energy shifts to the electronic structure. In order to establish such a methodology, the $\mathrm{CaO}(100)$ surface to bulk core-level binding energy shifts have been studied with Hartree-Fock and density-functional theory methods using both cluster and periodic slab models. The shifts obtained from the different theoretical methods are compared with each other and with data from synchrotron-based x-ray photoelectron spectroscopy (XPS) measurements. With a common approximation for the slab model treatment of XPS, the predicted binding energy shifts are seriously in error. The origin of the error is identified as arising from a flawed treatment of the surface atom binding energies, and a method for correcting the failure is presented.
\end{abstract}

DOI: 10.1103/PhysRevB.100.115419

\section{INTRODUCTION}

The difference of the core-level binding energies (BEs) of atoms in the bulk and at the surface of solids, the surface corelevel shifts (SCLSs), has been extensively studied for metal surfaces using experimental and theoretical methods [1]. The different approaches to the calculation and the interpretation of the significance of SCLSs have been reviewed by Egelhof [1] and by Bagus et al. [2]. In contrast to the wealth of information available for metal surfaces, our previous SCLS study of $\mathrm{MgO}(100)$ [3] is the only such study available for oxide surfaces. This is surprising since surface core-level shifts are intimately correlated with the electronic and geometric properties of surface atoms, both of which determine the catalytic properties of the surface. However, to use the SCLSs to infer these properties of oxides, it is necessary to have reliable theoretical methods to predict the SCLSs of oxides.

If the bulk and surface atoms of an oxide are considered to be ideal ions, the difference of the Madelung potentials of

\footnotetext{
*Present address: Department of Physics, University of Texas, Austin, TX 78712, USA.

${ }^{\dagger}$ Present address: Skolkovo Institute of Science and Technology, Skolkovo Innovation Center, 3 Nobel Street, 143026 Moscow, Russia.

¥Present address: Abteilung Grenzflächenwissenschaft, FritzHaber-Institut der Max-Planck-Gesellschaft, Faradayweg 4-6, 14195 Berlin, Germany.

${ }^{\S}$ Present address: Faculty of Chemical, Environmental and Biological Science, Technology, Dalian University of Technology, Dalian 116024, China.

"kuhlenbeck@fhi-berlin.mpg.de
}

bulk and surface atoms will determine the SCLS. In an octahedral oxide, a bulk ion has six nearest counterion neighbors, whereas a surface ion has only five such neighbors. Therefore, the magnitude of the Madelung potential for surface ions will be smaller than for bulk ions. As the Madelung potential raises anion and lowers cation core-level BEs, the surface anion and cation core-level BEs will be lower and higher than those in the bulk, respectively [3]. For cubic oxides with lattice constants of 4-5 $\AA$, e.g., $\mathrm{MgO}$ or $\mathrm{CaO}$, the magnitude of the Madelung potential at surface anion and cation sites is different from that in the bulk by $\sim 1 \mathrm{eV}$. This would certainly lead to an observable BE shift. Our previous experimental and theoretical study of the SCLSs of $\mathrm{MgO}$ [3], which is an ideal ionic insulator [3,4], used $a b$ initio Hartree-Fock (HF) wave functions (WFs) for cluster models of the bulk and the (100) surface. It was found that the $\operatorname{Mg}(2 p)$ SCLS is close to the difference arising from the surface and bulk Madelung potentials. On the other hand, the SCLS of $\mathrm{O}(1 s)$ was near zero, indicating that other electronic effects must cancel out the difference in the oxygen surface and bulk Madelung potentials. Further, it seemed reasonable that this cancellation should hold also for other dominantly ionic oxides, including $\mathrm{CaO}$.

Thus, it was puzzling that preliminary calculations showed that the $\mathrm{CaO}(100)$ SCLSs for the $\mathrm{Ca}(2 p)$ and $\mathrm{O}(1 s)$ were of comparable magnitude, $\sim 0.5 \mathrm{eV}$, but of opposite sign, quite different from the SCLSs for $\mathrm{MgO}(100)$. These results were based on density-functional theory (DFT) calculations for a periodic slab model using a conventional DFT approach to calculate core-level BEs, the Slater-Janak transition state approximation; see Ref. [5]. These preliminary DFT results raised several important questions related to the electronic structure and properties of $\mathrm{CaO}$ and to the different theoretical 
approaches-periodic DFT, used to study $\mathrm{CaO}$, and $a b$ initio $\mathrm{HF}$ WF theory for embedded clusters used to study $\mathrm{MgO}$. Since it was known that the HF WFs for cluster models correctly described the anion and cation SCLSs for $\mathrm{MgO}$, one possibility was that $\mathrm{CaO}$ was not nearly an ideal ionic material as $\mathrm{MgO}$. There was also the possibility that the periodic DFT slab model theory failed to correctly describe the shifts between the BEs of bulk and surface ions. This issue was investigated using two approaches-(1) an experimental determination of the $\mathrm{CaO}(100)$ SCLSs with XPS (x-ray photoelectron spectroscopy), and (2) using DFT densities for embedded clusters to check whether the BE shifts would be different from those obtained with ab initio HF WFs. When experiment, cluster model WF theory, and cluster model DFT theory all gave qualitatively the same SCLSs for $\mathrm{CaO}(100)$, attention was turned to identify and correct the origin of the failure of the slab model DFT treatment. The failure was identified as an artifact of an approximation to retain neutral supercells when using a periodic model to compute core-level BEs.

\section{COMPUTATIONAL AND EXPERIMENTAL}

The periodic slab model has been broadly used to model core-level BEs as encountered with XPS [6-12]. In the present paper, we consider a standardly used method where a fractional electron is promoted to the conduction band of the slab $[6,7,9,10,12]$ and we describe this as the "conventional" method. We have identified an error in BE shifts that may arise with this conventional method and further we have identified the reason for this error in terms of a localization of the excited fractional electron. It is important that people who use the conventional method to neutralize the supercell be aware of this problem since it may arise in other situations beyond the one that we have explicitly treated. In addition, we have proposed and tested a method to correct the localization problem for slab model BEs and BE shifts. The method has the special advantages of simplicity and the fact that it can be directly used with standard computational methods and programs for slab model calculations. The approach can be used as an alternative to or in conjunction with more advanced approaches to modeling charged systems of reduced dimensionality [13-15].

Core-level XPS measurements were performed at the UE56/2 PGM 1 beamline at Helmholtz-Zentrum Berlin. $\mathrm{CaO}(100)$ films, of $\sim 11 \mathrm{~nm}$ thickness, were prepared on a $\mathrm{Pt}(100)$ single crystal cleaned by cycles of $\mathrm{Ar}^{+}$ion sputtering, oxidation, and annealing until a sharp low-energy electron diffraction $($ LEED) pattern of the $(5 \times 20)$ reconstruction [16] was observed. XPS peak fitting was performed using the CASA XPS software. [17] Prior to fitting, a Shirley background [18] was subtracted from the data. Details of the sample preparation and the XPS measurements are given in the Supplemental Material [19] (see also Refs. [20-26]).

HF WFs and DFT densities were determined with embedded cluster models of $\mathrm{CaO}(100)$. The clusters were chosen based on our previous work on the SCLSs of $\mathrm{MgO}(100)$ [3], where it has been shown that clusters of the size used in this work will provide reliable descriptions of the surface and bulk electronic structure of $\mathrm{MgO}$ including the $\mathrm{Mg}$ and O SCLSs. Following this approach for the Ca BEs, the bulk (surface) clusters were point-charge embedded $\mathrm{CaO}_{6} \mathrm{Ca}_{18}$ $\left(\mathrm{CaO}_{5} \mathrm{Ca}_{13}\right)$ clusters, and for the $\mathrm{O}$ BEs, they were pointcharge embedded $\mathrm{OCa}_{6} \mathrm{O}_{18} \mathrm{Ca}_{38}\left(\mathrm{OCa}_{5} \mathrm{O}_{13} \mathrm{Ca}_{25}\right)$ clusters. The single central atom in each cluster was the atom whose core shell was ionized. Graphical views of the clusters are shown in the SM [19], where also the logic of the choice of them is discussed. For the slab model DFT calculations, five-layer slabs of the $(100)$ surface of $\mathrm{CaO}$, denoted $(3 \times 3 \times 5)$, were constructed from a cubic primitive bulk unit cell. The geometry of the slab was fully relaxed to take into account the surface reconstruction leading to an effective lattice constant for $\mathrm{CaO}$ of $a_{0}=4.834 \AA$ compared to the experimental value of $a_{0}=4.81 \AA$ [26]. Further parameters of the slab are given in the SM [19].

Separate calculations of the HF WFs and the DFT densities were carried out for the initial and final (core ionized) configurations. Details of the calculations are given in the SM [19]. The initial state configuration for all cluster and slab models is closed shell and can be viewed as being composed of $\mathrm{Ca}^{2+}$ cations and $\mathrm{O}^{2-}$ anions, possibly modified by covalent mixing. Core ions had an electron removed from either $\mathrm{Ca}(2 p)$ or $\mathrm{O}(1 s)$. Surface and bulk BEs were computed in the slab model approach for slabs with a core ion at the surface or in the third layer, respectively. For the cluster model calculations different clusters for bulk and surface ionizations were used (see SM [19]). In these calculations, the total BE, denoted $\mathrm{BE}(\triangle \mathrm{SCF})$, is the difference of the variationally optimized, self-consistent field or SCF, initial state energy, E(initial), and the variationally optimized energy of the core ion, $E($ ion $)$ with $B E(\triangle \mathrm{SCF})=E($ ion $)-E($ initial $)$.Within DFT, the Slater-Janak transition state approximation [9,27], where the BE is taken as the Kohn-Sham orbital energy of the ionized shell with an occupation reduced by 0.5 electrons, gives a good approximation to $\mathrm{BE}(\triangle \mathrm{SCF})$ [28]. For the DFT cluster model calculations where both $\mathrm{BE}(\triangle \mathrm{SCF})$ and the Slater-Janak transition state approximation can be used, the two BEs will be compared. However, for the slab models, it is not possible to remove an electron from the supercell because of the long-range Coulomb interactions between the charged supercells. To avoid this difficulty, the 0.5 electron removed from the core shell is added into the conduction band which allows the supercell to be neutral. This approximation is regularly used for the BEs of conductors but we have discovered that it leads to artifacts for ionization of core levels in ionic crystals. We have been able to avoid these artifacts with the use of the virtual-crystal approximation (VCA) [29,30], where the supercell is made neutral not by adding an electron to the conduction band but by removing small fractions of charge from atoms in the supercell.

\section{RESULTS AND DISCUSSION}

$\mathrm{Ca}\left(2 p_{3 / 2}\right)$ photoemission spectra recorded at normal emission for photon energies from 450 to $750 \mathrm{eV}$ are shown in Fig. 1(a). The peaks can be fit well with two Voigt functions (70\% Gaussian), with the main peak at $345.7 \mathrm{eV}$ and another, smaller peak at $0.6 \mathrm{eV}$ higher binding energy. The relative intensity of the smaller peak increases from $8 \%$ to $31 \%$ as the surface sensitivity is increased by decreasing the photon energy. $\mathrm{O}(1 s)$ spectra at normal emission for photon energies 


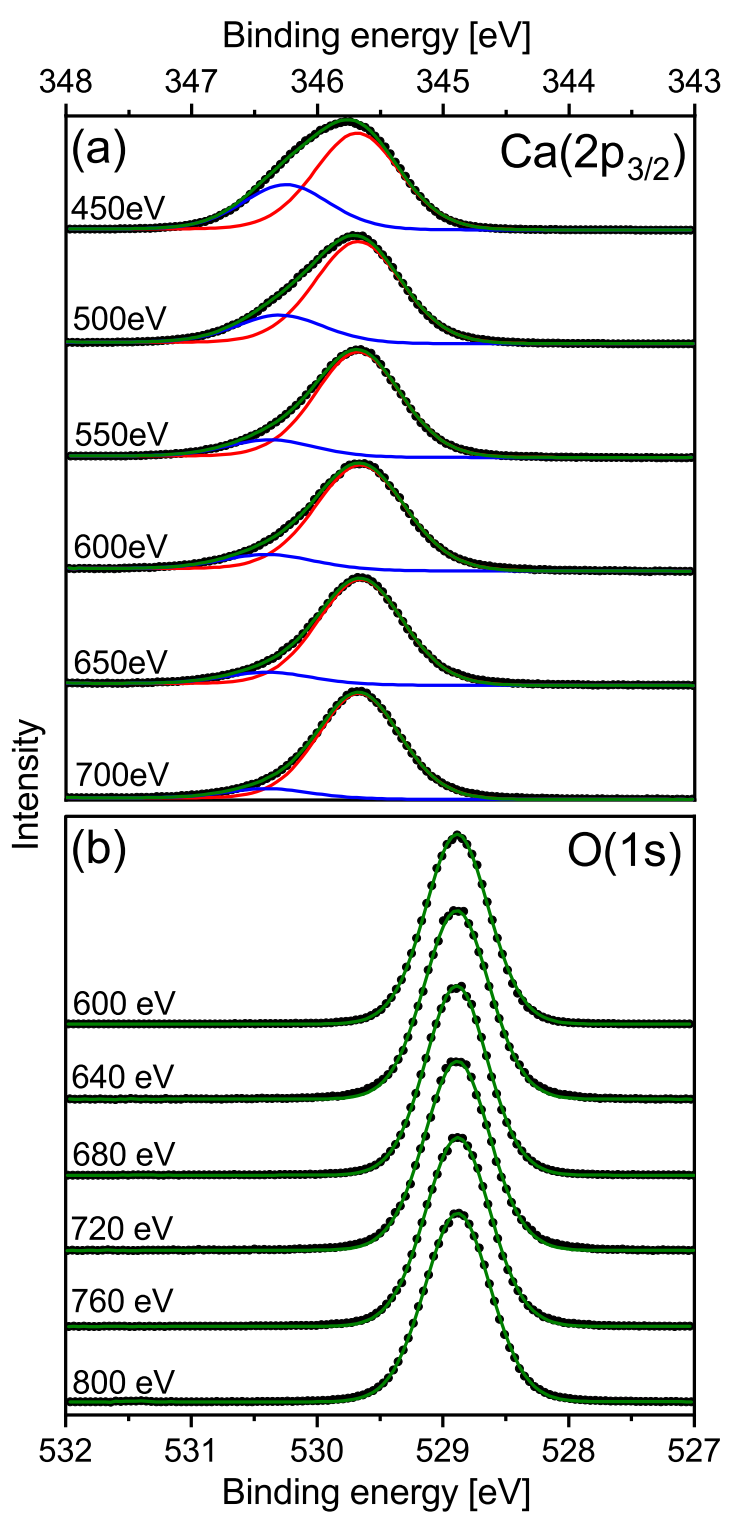

FIG. 1. XPS spectra of the $\mathrm{Ca}\left(2 p_{3 / 2}\right)$ [top panel, (a)] and $\mathrm{O}(1 s)$ [bottom panel, (b)] core levels with the surface sensitivity varied by changing the photon energy. Fits are shown as blue (surface core level), red (bulk core level), and olive (sum) curves for the $\mathrm{Ca}\left(2 p_{3 / 2}\right)$ level. For the $\mathrm{O}(1 s)$ spectra just a single fit function was used (olive color).

from 600 to $800 \mathrm{eV}$ are shown in Fig. 1(b). The $\mathrm{O}(1 s)$ peak in each case was fitted with a single Voigt peak. Peaks due to hydroxyl were not observed. It was not possible to resolve a surface component from the bulk $\mathrm{O}(1 s)$ peak. Since the surface is free of defects and hydroxyls, it is concluded that the SCLS for $\mathrm{O}(1 s)$ must be less than $\sim 0.1 \mathrm{eV}$.

The $\mathrm{Ca}(2 p)$ and $\mathrm{O}(1 s)$ SCLSs of $\mathrm{CaO}(100)$ obtained with the $a b$ initio HF and DFT calculations for the cluster models and the periodic slab model are compared with experiment in Table I. For the surface $\mathrm{Ca}(2 p) \mathrm{BE}$, the ionization of the $2 p$ component normal to the surface is used; however, the HF calculation shows that the splitting of the normal and parallel $\mathrm{Ca}(2 p) \mathrm{BEs}$ is only $0.01 \mathrm{eV}$. The SCLSs computed using either $\triangle \mathrm{SCF}$ or Slater-Janak, denoted hereafter as SJ,
TABLE I. CaO SCLSs, in eV, obtained by different theoretical methods and compared with experiment; see text for definitions of the methods.

\begin{tabular}{lcc}
\hline \hline & $\mathrm{Ca}(2 p)$ & $\mathrm{O}(1 s)$ \\
\hline HF cluster- $\Delta \mathrm{SCF}$ & +1.00 & +0.05 \\
DFT cluster- $-\mathrm{SCF}$ & +1.04 & +0.17 \\
DFT cluster-SJ & +1.09 & +0.15 \\
Conventional slab-SJ & +0.47 & -0.42 \\
VCA slab-SJ & +0.78 & -0.04 \\
Measured (XPS) & +0.6 & $|\mathrm{SCLS}|<0.1$ \\
\hline \hline
\end{tabular}

and the other methods are listed in the table. For the slab model SCLSs, the SJ approximation is used but two different approaches are taken to keep the periodic supercell neutral. One method, described as conventional, is to promote the 0.5 core electron into a vacant level in the conduction band; see Refs. [6,7,9,10,12]. The second method is based on the VCA [29-32] where the neutrality of the supercell is maintained by modifications of the atomic charges of other atoms in the supercell. These two slab model approaches are compared in detail below.

The HF and DFT cluster results are reasonably similar with the $\mathrm{Ca}(2 p)$ SCLS being large, $\sim 1.0 \mathrm{eV}$, and the $\mathrm{O}(1 s)$ SCLS being small, $\sim 1.0 \mathrm{eV}$. Since the HF and DFT calculations used precisely the same cluster, the good agreement is hardly surprising and the small differences reflect different $\mathrm{HF}$ and DFT approximations. Furthermore, the $\triangle \mathrm{SCF}$ and SJ values of the SCLSs are reasonably similar. The agreement of the $\triangle \mathrm{SCF}$ and the approximate SJ SCLSs justifies the use of the SJ approximation for the slab model SCLSs. The cluster model SCLSs are consistent with experiment, being large for $\mathrm{Ca}(2 p)$ and small for $\mathrm{O}(1 s)$. In particular, for reasons that will be mentioned briefly below and discussed in more detail in Ref. [33], it is expected that the cluster results will give a larger $\mathrm{Ca}(2 p)$ SCLS than measured. On the other hand, the conventional slab model SCLSs are quite different from both the cluster model theory and the measured SCLSs: the $\mathrm{Ca}(2 p)$ and $\mathrm{O}(1 s)$ SCLSs are $\sim 0.5 \mathrm{eV}$ smaller than the HF and DFT cluster values, an indication that the surface core-level BEs given by the slab model are too small by $0.5 \mathrm{eV}$.

A possible origin for the reduction of the surface atom BEs is that the additional 0.5 electron added to the conduction band for a surface ion is not delocalized over the slab layers but is localized around the ionized surface atom. The effect of such a localized charge would be to lower the core-level BE. It would not require a very large localization to lower a core-level $\mathrm{BE}$ by only $0.5 \mathrm{eV}$ [34]. This localization is possible for the surface ions where diffuse $\mathrm{Ca}(4 s)$ and $\mathrm{Ca}(4 p)$ orbitals and the $\mathrm{O}(3 s)$ and $\mathrm{O}(3 p)$ orbitals are available in the region above the surface. This is in contrast to the bulk where these Rydberg orbitals are quenched, thus reducing the possibility of localization. The distribution of the 0.5 electron for the $\mathrm{O}(1 s)$ core-excited bulk and surface states is shown graphically in Fig. 2. It is clear that the added electron for the surface $\mathrm{O}(1 s)$ ionization is not delocalized throughout the slab but is strongly localized near the $\mathrm{O}$ anion where the fractional electron is removed from the core. For the bulk $\mathrm{O}(1 s)$ 

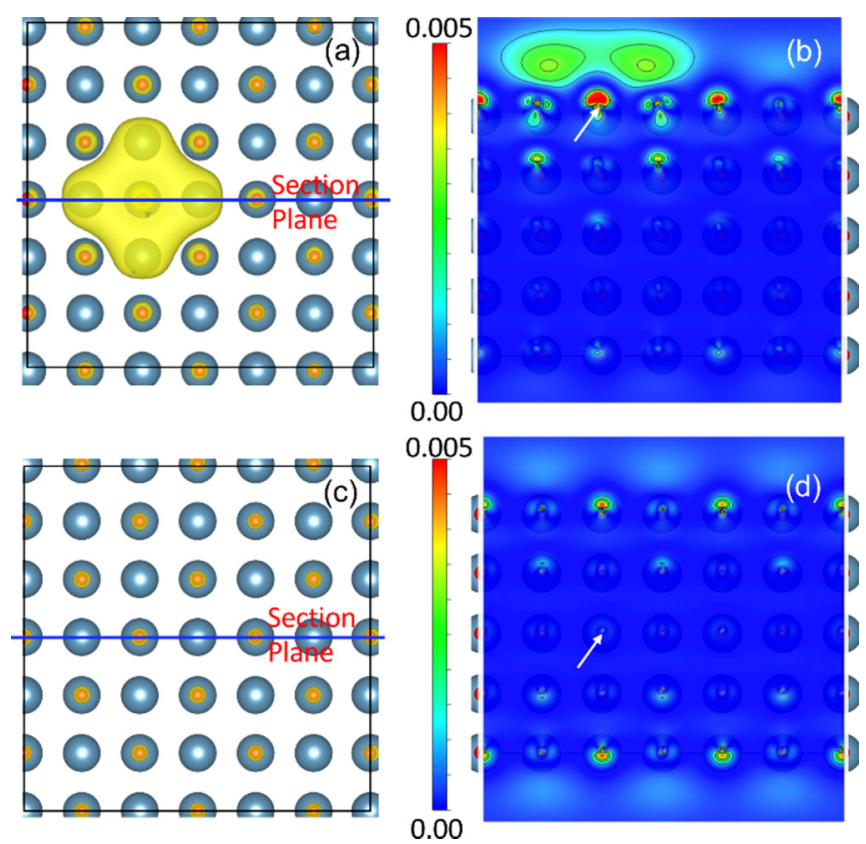

FIG. 2. Cross sections of the density of the 0.5 electron in the conduction band for the slab model of $\mathrm{CaO}(100)$, compensating an $\mathrm{O}(1 s)$ core hole [marked with arrows in panels $(\mathrm{b}, \mathrm{d})]$. The $(\mathrm{a}, \mathrm{b})$ panels show the density for a core hole at the surface and the $(c, d)$ panels are for a core hole in the middle (third) layer of the slab. (a,c) are in top view and $(b, d)$ are in side view. The units of the color scale are electrons per $\AA^{3}$.

ionization, the added electron is much more delocalized although the distribution of the density is not entirely uniform and the density is somewhat localized around the surface of the slab. It is expected that a similar localization will be found for the added 0.5 electrons in the case of a surface $\mathrm{Ca}(2 p)$ ionization. These localizations are consistent with the hypothesis that the error of the conventional slab model SCLSs arises from the localization of the added surface density that was used to bring the slab supercell back to being neutral.

It is possible to uniformly distribute the excited 0.5 electron over the supercell which has been done for periodic models of the bulk [5]. However, for the slab model most of the charge would be in the vacuum which is not physically reasonable. A way out would be to restrict the charge distribution to the region within the slab. However, the choice of the region, and the way it is truncated, smooth or abrupt, remain arbitrary and can affect the electronic structure.

The VCA approach $[29,31,32]$ avoids the issues described above. This approach was originally developed for modeling solid solutions but can be adapted to simulate doping and provide charge compensation that does not perturb the valence electrons. $[29,30]$ When half an electron is removed from a cation or an anion the excess positive charge in the supercell is compensated by reducing the charge of the nuclei. We restrict the modification of the nuclear charges to the complementary sublattice (i.e., to $\mathrm{Ca}$ nuclei for an $\mathrm{O}$ core hole, and vice versa). Thus, for $\mathrm{O}(1 s)$ ionization, each of the $90 \mathrm{Ca}$ atoms in the supercell of the $3 \times 3 \times 5$ slab model is reduced by $0.5 / 90$ from $Z=20.0$ to $Z=19.994$. While this will change the total energy of the supercell, the changes will be similar for bulk and surface ionization. With the VCA approximation to maintain the neutrality of the supercell, the slab model SCLSs are now nearly consistent with experiment and with the HF and DFT cluster model results; see Table I. The VCA slab model Ca(2p) SCLS is closer to the experimental value than the cluster model SCLS, which is because the slab geometry has been optimized and includes the effects of surface reconstruction while the cluster geometry is fixed at the bulk geometry. The influence of the $\mathrm{CaO}(100)$ surface reconstruction on the SCLSs is examined in Ref. [33] where it is shown that this reconstruction leads to a reduction of the $\mathrm{Ca}(2 p)$ SCLS. The measured $\mathrm{Ca}(2 p)$ spectra also have contributions from the ionization of second layer $\mathrm{Ca}$ atoms which will have BEs intermediate between the calculated surface layer BEs and the bulk atom BE, which might affect the experimentally determined SCLS somewhat.

\section{CONCLUSIONS}

In conclusion, we have shown that cluster model calculations for the SCLSs of $\mathrm{CaO}(100)$ using HF WFs or DFT densities give good agreement with XPS measurements while the SCLSs obtained with conventional slab model methods do not. The errors for the conventional treatment in periodic slab model XPS calculations are due to the approximation of maintaining the neutrality of the supercell by adding a fraction of an electron to the conduction band. The failure arises from a localization of the fractional electron in the region near the ionized surface atom. This can be avoided using the VCA approach. Here, the neutrality of the supercell with the core-ionized atom is maintained by removing a small fraction of charge from the nuclei of the counterions in the supercell. With this, the $\mathrm{CaO}$ SCLSs are now large, $\sim 1.0 \mathrm{eV}$, for $\mathrm{Ca}(2 p)$ ionization, and small, $\sim 0 \mathrm{eV}$, for $\mathrm{O}(1 s)$ ionizationconsistent with both the ab initio HF and DFT cluster SCLSs, with the XPS measurements, and with previously obtained results for $\mathrm{MgO}(100)$.

We emphasize that the scope of the discussed failure is not restricted to SCLSs for $\mathrm{CaO}(100)$. The XPS final state is ionic by nature, which demands measures to compensate the charge in slab model calculations. As we show here, adding charge to the conduction band, which is a common method of charge compensation, may lead to erroneous results due to the interaction of the core hole with the added charge. Since the concern is for the participation of the added half an electron in the screening of the core hole, it is to be expected that this is a general issue for XPS binding energy shift calculations using periodic models, not just for SCLSs. Furthermore, XPS binding energy shift calculations using the slab model approach are common in the literature; Refs. [5,35-40] give representative examples of the use of slab models for XPS. We have shown that the VCA approach represents a safe way to avoid artifacts related to the screening by the added half an electron in the conduction band and we have shown that these artifacts can result in notable errors, $\sim 0.5 \mathrm{eV}$ in the present case. Since the screening of the core holes is different in metals and nonmetals, the artifact that we found for insulators might be less important for metals; however, this is an issue that needs to be investigated. 


\section{ACKNOWLEDGMENTS}

P.S.B. acknowledges support from the US Department of Energy, Office of Science, Office of Basic Energy Sciences, Chemical Sciences, Geosciences, and Biosciences (CSGB) Division through the Geosciences program at Pacific Northwest National Laboratory. E.D., H.K., and H.-J.F. acknowl- edge support by the DFG through their SFB 546, Transition Metal Oxide Aggregates. F.S. and C.P. thank the Cluster of Excellence "Engineering of Advanced Materials" for financial support and X.Z. acknowledges Prof. A. Selloni in Princeton and the NERSC facility for computation time (Grant No. DE-AC02-05CH11231). We thank HZB for the allocation of synchrotron radiation beam time.
[1] W. F. Egelhoff, Surf. Sci. Rep. 6, 253 (1987).

[2] P. S. Bagus, E. S. Ilton, and C. J. Nelin, Surf. Sci. Rep. 68, 273 (2013).

[3] C. J. Nelin, F. Uhl, V. Staemmler, P. S. Bagus, Y. Fujimori, M. Sterrer, H. Kuhlenbeck, and H.-J. Freund, Phys. Chem. Chem. Phys. 16, 21953 (2014).

[4] G. Pacchioni, C. Sousa, F. Illas, F. Parmigiani, and P. S. Bagus, Phys. Rev. B 48, 11573 (1993).

[5] Y. Fujimori, X. Zhao, X. Shao, S. V. Levchenko, N. Nilius, M. Sterrer, and H.-J. Freund, J. Phys. Chem. C 120, 5565 (2016).

[6] T. Aizawa, S. Suehara, S. Hishita, S. Otani, and M. Arai, Phys. Rev. B 71, 165405 (2005).

[7] M. V. Ganduglia-Pirovano, M. Scheffler, A. Baraldi, S. Lizzit, G. Comelli, G. Paolucci, and R. Rosei, Phys. Rev. B 63, 205415 (2001).

[8] D. Golze, J. Wilhelm, M. J. van Setten, and P. Rinke, J. Chem. Theory Comput. 14, 4856 (2018).

[9] S. Lizzit, A. Baraldi, A. Groso, K. Reuter, M. V. GandugliaPirovano, C. Stampfl, M. Scheffler, M. Stichler, C. Keller, W. Wurth et al., Phys. Rev. B 63, 205419 (2001).

[10] W. Olovsson, C. Göransson, L. V. Pourovskii, B. Johansson, and I. A. Abrikosov, Phys. Rev. B 72, 064203 (2005).

[11] T. Ozaki and C.-C. Lee, Phys. Rev. Lett. 118, 026401 (2017).

[12] Z. Zeng, X. Ma, W. Ding, and W. Li, Sci. China Chem. 53, 402 (2010).

[13] C. Freysoldt and J. Neugebauer, Phys. Rev. B 97, 205425 (2018).

[14] M. R. Jarvis, I. D. White, R. W. Godby, and M. C. Payne, Phys. Rev. B 56, 14972 (1997).

[15] C. A. Rozzi, D. Varsano, A. Marini, E. K. U. Gross, and A. Rubio, Phys. Rev. B 73, 205119 (2006).

[16] H. P. Bonzel, C. R. Helms, and S. Kelemen, Phys. Rev. Lett. 35, 1237 (1975).

[17] http://www.casaxps.com.

[18] D. A. Shirley, Phys. Rev. B 5, 4709 (1972).

[19] See Supplemental Material at http://link.aps.org/supplemental/ 10.1103/PhysRevB.100.115419 for details of the sample preparation and the XPS measurements.

[20] CLIPS is a program system to compute ab initio SCF and correlated wave functions for polyatomic systems. It has been developed based on the publicly available programs in the ALCHEMY package from the IBM San Jose Research Laboratory by P. S. Bagus, B. Liu, A. D. McLean, and M. Yoshimine.

[21] V. Blum, R. Gehrke, F. Hanke, P. Havu, V. Havu, X. Ren, K. Reuter, and M. Scheffler, Comput. Phys. Commun. 180, 2175 (2009).

[22] J. Q. Broughton and P. S. Bagus, Phys. Rev. B 36, 2813 (1987).

[23] H. M. Evjen, Phys. Rev. 39, 675 (1932).

[24] A. V. Krukau, O. A. Vydrov, A. F. Izmaylov, and G. E. Scuseria, J. Chem. Phys. 125, 224106 (2006).

[25] J. P. Perdew, K. Burke, and M. Ernzerhof, Phys. Rev. Lett. 77, 3865 (1996).

[26] R. W. G. Wyckoff, Crystal Structures (Wiley, New York, 1963).

[27] J. F. Janak, Phys. Rev. B 18, 7165 (1978).

[28] D. P. Chong, Chem. Phys. Lett. 232, 486 (1995).

[29] N. A. Richter, S. Sicolo, S. V. Levchenko, J. Sauer, and M. Scheffler, Phys. Rev. Lett. 111, 045502 (2013).

[30] O. Sinai and L. Kronik, Phys. Rev. B 87, 235305 (2013).

[31] L. Vegard, Z. Phys. 5, 17 (1921).

[32] M. Scheffler, Physica B+C (Amsterdam) 146, 176 (1987).

[33] P. S. Bagus, C. J. Nelin, X. Zhao, S. V. Levchenko, E. Davis, X. Weng, C. Papp, H. Kuhlenbeck, and H.-J. Freund (unpublished).

[34] P. S. Bagus, E. S. Ilton, and C. J. Nelin, Catal. Lett. 148, 1785 (2018).

[35] A. Baby, G. Fratesi, S. R. Vaidya, L. L. Patera, C. Africh, L. Floreano, and G. P. Brivio, J. Phys. Chem. C 119, 3624 (2015).

[36] T. Kendelewicz, S. Kaya, J. T. Newberg, H. Bluhm, N. Mulakaluri, W. Moritz, M. Scheffler, A. Nilsson, R. Pentcheva, and G. E. Brown, J. Phys. Chem. C 117, 2719 (2013).

[37] M. Setvin, X. Shi, J. Hulva, T. Simschitz, G. S. Parkinson, M. Schmid, C. Di Valentin, A. Selloni, and U. Diebold, ACS Catal. 7, 7081 (2017).

[38] Q. T. Trinh, K. Bhola, P. N. Amaniampong, F. Jérôme, and S. H. Mushrif, J. Phys. Chem. C 122, 22397 (2018).

[39] H. Wang, S. V. Levchenko, T. Schultz, N. Koch, M. Scheffler, and M. Rossi, Adv. Electron. Mater. 5, 1800891 (2019).

[40] R. Zhang, A. J. Hensley, J.-S. McEwen, S. Wickert, E. Darlatt, K. Fischer, M. Schöppke, R. Denecke, R. Streber, and M. Lorenz et al., Phys. Chem. Chem. Phys. 15, 20662 (2013). 